\title{
Summary of the CIC Recommendations for zoster immunization programs
}

\author{
Canadian Immunization Committee ${ }^{1 \star}$
}

*Correspondence to: CIC Secretariat (cic-cci@phac-aspc.gc.ca)

\section{Introduction}

Zoster infection results from the reactivation of the varicella zoster virus and typically occurs many years after primary infection. Varicella zoster virus is associated with chicken pox in childhood and zoster infection in adulthood. The overall incidence of zoster is increasing in Canada, the elderly being the most susceptible. Immunocompromised persons are also an increased risk of zoster infection. Zoster infection is characterized by neuropathic pain and a dermatomal rash, and carries a lifetime risk of $15 \%-28 \%$ in Canada. Complications of zoster infection occur in $13 \%-40 \%$ of cases. The most frequent complication is pain, including post-herpetic neuralgia, which is significant pain persisting longer than 90 to 120 days after rash onset. Post-herpetic neuralgia has a significant adverse impact on quality of life and occurs in one-third of zoster cases over the age of 60 , the risk increasing with age. On the basis of scientific evidence, the National Advisory Committee on Immunization recommends zoster vaccine for the prevention of zoster and its complications in persons 60 years and older without contraindications and recommends that it may be used in those aged 50-59 years. Zoster vaccine is currently not publicly funded in Canada. This document is a brief summary of the full report of the Canadian Immunization Committee (CIC) zoster recommendations (1). Recommendations for Zoster Immunization Programs

\section{Objective}

To consider whether a population-based, publicly funded zoster vaccine program can be recommended.

\section{Approach}

The CIC statement considered the disease characteristics and burden of illness; vaccine safety and efficacy; immunization strategies and programs; cost-effectiveness; feasibility and acceptability; ability to evaluate a zoster immunization program; outstanding research questions; and equity, ethical and political considerations.

\section{Findings}

Zostava ${ }^{\circledR}$ is a live, attenuated zoster vaccine approved for use in Canada in those aged 50 years and older without contraindications. The efficacy and safety of Zostavax ${ }^{\circledR}$ have been demonstrated through the Shingles Prevention Study and related substudies; the duration of protection beyond 7 years is uncertain, and studies to 10 years are in progress.

The delivery of zoster vaccine can be optimized by coadministration with influenza and pneumococcal vaccines given to older adults. A review of cost-effectiveness analyses from 12 publications from seven countries indicates that cost-effectiveness is maximized when zoster vaccine is given to adults between the ages of 60-69. The feasibility of a zoster immunization program may be affected by the need for the present formulation to be frozen. However, it is currently projected that a refrigerator-stable formulation will be available in Canada starting March 2014, which will likely improve feasibility.

Evaluation of a zoster immunization program will be complex, and many factors must be considered, such as the availability of information systems to 1) measure coverage, utilization and quality; 2) monitor reduction of disease incidence, complications, sequelae and mortality; 3 ) monitor adverse events associated with vaccine administration; and 4) link health outcomes databases, immunization registries and population registries. Ongoing and planned research includes studies involving older adults and immunocompromised individuals, studies with 
an inactivated heat-stable vaccine, and studies of the duration of protection. The long-term impact of varicella vaccine programs on zoster epidemiology should be studied further, along with vaccine effectiveness as demonstrated by a reduction in disease rates, burden of illness and rates of post-herpetic neuralgia.

\section{Recommendations}

The CIC recommends routine offering of zoster vaccine to immunocompetent adults aged 60 to 65 years and older without contraindications on the basis of the epidemiology of varicella zoster virus, zoster vaccine characteristics, disease modeling and economic analysis, as well as on the feasibility and acceptability of zoster immunization programs. There are no national goals for zoster prevention in Canada, and development of such goals would guide the planning of organized programs. In the absence of a publicly funded program, the vaccine is available for private purchase.

\section{Reference}

(1) Canadian Immunization Committee. Recommendations for zoster immunization programs. 2013.

http://publications.gc.ca/site/eng/464259/publication.html

${ }^{1}$ Note: The Canadian Immunization Committee $(\mathrm{CIC})$ provides operational and technical advice related to immunization policies and programs in Canada. $\mathrm{CIC}$ consists of representatives from provincial and territorial jurisdictions, the National Advisory Committee on Immunization ( $\mathrm{NACl}$ ), the American Advisory Committee on Immunization Practices (ACIP) and Health Canada's Biologics and Genetic Therapies Directorate (BGTD), Marketed Health Products Directorate (MHPD), and First Nations and Inuit Health Branch. 